\title{
Quality of life in patients with juvenile arthritis: adalimumab might make a difference
}

\author{
Rina Denisova, Ekaterina Alexeeva, Saniya Valieva, Tatyana Bzarova, Elena Mitenko ${ }^{*}$, Tatyana Sleptsova, \\ Kseniya Isayeva \\ From 18th Pediatric Rheumatology European Society (PReS) Congress \\ Bruges, Belgium. 14-18 September 2011
}

\section{Introduction}

Juvenile arthritis (JA) leads to premature impairment of pediatric physical health, psychological and social limitations, that considerably worsen health related quality-oflife of patients. That is why improvement of health related quality-of-life is one of the main aims of treatment of patients with juvenile arthritis.

\section{Purpose}

Evaluate influence of adalimumab therapy on HRQOL of children with different forms of juvenile arthritis.

\section{Material and methods}

There were examined 66 children (median age $10(3 ; 16)$ years). From them there were 44 girls, 23 boys, 23 persons with oligoarticular juvenile idiopathic arthritis (JIA), 30-with polyarticular JIA, 3-with systemic JIA and 10 - with Juvenile Enthesitis related arthritis. 58 (88\%) children were treated with adalimumab for more than a year in the dose of $40 \mathrm{mg}$ every 2 weeks Evaluation of HRQOL was performed by the help of questionnaires PedsQL Generic Core Scale (PedsQL GCS), PedsQL Rheumatology Module (PedsQL RM).

\section{Results}

HRQOL of children with JA before the conducted therapy in all scales of questionnaire PedsQL GCS was considerably lower in comparison with population norm $(p<0,001)$. To week 54 of infliximab therapy there was detected statistically reliable increase of HRQOL values in all scales of the questionnaires $(p<0,001)$. By self report of the questionnaires PedsQL RM by the scale "pain and hurt" HRQOL Me increased from 37 to 100, by the scale "daily activities" - from 75 to 100 , by the scale "treatment" - from 45 to 74 , by the scale "worry" from 45 to 65 , by the scale "communication" from 55 to 75. By proxy report from 25 to 100 , from 55 to 100 , from 30 to 60 , from 35 to 62 , from 50 to 70 , respectively. By self report of the questionnaire PedsQL GCS total score of HRQOL increased from 47 to 79,23 , by the scale of physical health - from 43 to 86,15 , by the scale of emotional functioning - from 45 to 72,16 and by the scale of social functioning - from 50 to 79,38 . By proxy report from 38 to 75,14 , from 37 to 81 , from 35 to 68,28 , from 40 to 72,69 , respectively. To the year of receiving the therapy by self report health related quality-of-life differed from population norm only by the scale of social functioning $(\mathrm{p}<0,01)$, and by proxy report HRQOL was lower only by the scales of emotional and social functioning $(\mathrm{p}<0,001)$.

\section{Conclusion}

Adalimumab administration in pediatric rheumatologic practice increases physical, psychological, social adaptation of patients, allows changing diagnosis of such a severe disabling disease, as JA, stopping steadily progressing disease course and preventing severe disability in such tender age.

Published: 14 September 2011

doi:10.1186/1546-0096-9-S1-P40

Cite this article as: Denisova et al:: Quality of life in patients with juvenile arthritis: adalimumab might make a difference. Pediatric Rheumatology 2011 9(Suppl 1):P40. 